Optical focusing through biological tissue and tissue-mimicking phantoms up to 9.6 centimeters thick with digital optical phase conjugation

Yuecheng Shen

Yan Liu

Cheng $\mathrm{Ma}$

Lihong V. Wang 


\title{
Optical focusing through biological tissue and tissue- mimicking phantoms up to 9.6 centimeters thick with digital optical phase conjugation
}

\author{
Yuecheng Shen ${ }^{1,2, \dagger}$, Yan Liu ${ }^{1,2, \dagger}$, Cheng $\mathrm{Ma}^{2}$, and Lihong V. Wang ${ }^{1,2 *}$ \\ ${ }^{1}$ Optical Imaging Laboratory, Department of Medical Engineering, California Institute of \\ Technology, Pasadena, California 91125, USA \\ ${ }^{2}$ Department of Biomedical Engineering, Washington University, Saint Louis, Missouri 63130, \\ USA \\ ${ }^{\dagger}$ Equal contribution*Corresponding author: LVW@,caltech.edu
}

\begin{abstract}
Optical phase conjugation (OPC) based wavefront shaping techniques focus light through or within scattering media, which is critically important for deep-tissue optical imaging, manipulation, and therapy. However, to date, the sample thicknesses used in wavefront shaping experiments have been limited to only a few millimeters or several transport mean free paths. Here, by using a long-coherence-length laser and an optimized digital OPC system that efficiently delivers light power, we focused $532 \mathrm{~nm}$ light through tissue-mimicking phantoms up to $9.6 \mathrm{~cm}$ thick, as well as through ex vivo chicken breast tissue up to $2.5 \mathrm{~cm}$ thick.
\end{abstract}

Keywords: Focusing light through scattering media, adaptive optics, wavefront shaping, optical phase conjugation, holographic interferometry, turbid media.

\section{INTRODUCTION}

Focusing light through or inside scattering media such as biological tissue is crucially important to deep tissue noninvasive optical imaging, manipulation, and therapy. However, because of the inherent refractive index inhomogeneity in biological tissue, direct light focusing by lenses beyond $\sim 1 \mathrm{~mm}$ deep is infeasible ${ }^{1,2}$. To overcome this optical diffusion limit, wavefront shaping techniques are being actively developed in recent years. By shaping the wavefront of the incident light, optical focusing can be achieved deep inside or through scattering media. There are several methods to determine the optimum wavefront, including feedback-based wavefront shaping ${ }^{3}$, transmission matrix measurement ${ }^{4,5}$, and optical time reversal/optical phase conjugation (OPC) ${ }^{6}$. However, no matter which method was used, the thicknesses of the samples used in previous studies were limited to only a few millimeters or several transport mean free paths $\left(l_{\mathrm{t}}^{\prime}\right)$, which are still relatively small for many preclinical and clinical applications. For example, using the feedback-based wavefront shaping method, Stockbridge et. al. demonstrated light focusing through 5 $\mathrm{mm}$ thick chicken tissue ${ }^{7}$. Using an analog OPC system based on a photorefractive crystal, McDowell et. al. demonstrated light focusing through $7 \mathrm{~mm}$ thick chicken tissue ${ }^{8}$. Recently, we demonstrated light focusing through 4 $\mathrm{mm}$ thick chicken tissue with a full-polarization digital OPC system (DOPC) ${ }^{9}$. The laser wavelengths were $532 \mathrm{~nm}$ in all three experiments. Since the principle of wavefront shaping does not impose an upper bound on either the sample thickness or the number of scattering events that can be tolerated, a natural question is whether we can focus light through a thicker sample.

To answer this question, in this work, we explored the experimental limit of the thickness of biological tissue and tissue-mimicking phantoms through which light can be focused, using the wavefront shaping methods. Firstly, we discussed the practical considerations that may restrict the sample thickness, including an inadequate laser coherence length, an insufficiently strong light signal, and a short speckle correlation time. With those considerations in mind, we designed and optimized the DOPC system and experimentally demonstrated light focusing through centimeter-thick biological tissue and tissue-mimicking phantoms. Specifically, we focused light through chicken breast tissue with

Adaptive Optics and Wavefront Control for Biological Systems III, edited by Thomas G. Bifano, Joel Kubby, Sylvain Gigan, Proc. of SPIE Vol. 10073, 100730G · C 2017 SPIE · CCC code: 1605-7422/17/\$18 · doi: 10.1117/12.2251052 
thicknesses up to $2.5 \mathrm{~cm}$ and through tissue-mimicking phantoms with thicknesses up to $9.6 \mathrm{~cm}^{10}$. The nearly $10 \mathrm{~cm}$ penetration has never been achieved before by any optical focusing technique.

\section{PRACTICAL CONSIDERATIONS AND SYSTEM DESIGN}

\subsection{Laser Coherence Length requirement}

We first discuss the requirement on the laser coherence length. As shown in Fig. 1, due to scattering, photons can take different paths to reach a target location (marked by the red dot). To make the photons propagating through different optical paths constructively interfere and form a focus at the target location, the laser coherence length should be longer than the optical path-length difference inside the scattering medium. To estimate the optical path-length difference for photons passing through a scattering medium with a thickness of $L$, we adopted the diffusion equation below ${ }^{11}$,

$$
\phi(r, t>L / c)=\frac{c}{(4 \pi D c t)^{3 / 2}} \exp \left(-\frac{\left(L-l_{t}{ }^{\prime}\right)^{2}}{4 D c t}\right),
$$

which tells us the distribution of the fluence rate as a function of photon arrival time. Here, $c$ is the speed of light in the scattering medium, $D$ is the diffusion coefficient, and $l_{\mathrm{t}}$ ' is the transport mean free path. We assume the absorption is negligibly weak for simplicity.

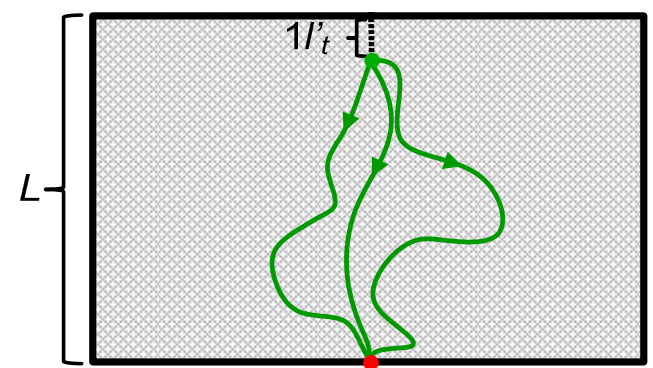

Figure 1. Schematic of photon paths to reach the location denoted by the red dot in a scattering medium with thickness $L$.

With this distribution, we can approximate the optical path length difference by multiplying the full width at half maximum of the time delay with the speed of light inside the scattering medium:

$$
\frac{\left(L-l_{t}^{\prime}\right)^{2}}{6 D}\left[\frac{1}{W_{-1}\left(-0.5^{2 / 3} / \mathrm{e}\right)}-\frac{1}{W_{0}\left(-0.5^{2 / 3} / \mathrm{e}\right)}\right]=\frac{\left(L-l_{\mathrm{t}}\right)^{2}}{2.2 D},
$$

where $W(\cdot)$ is the multivalued Lambert-W function. For details, please refer to the reference ${ }^{10}$. From this expression, we can see that the optical path-length difference is not linear with the sample thickness, but is proportional to the thickness squared. As an example, when the thickness of the sample is $10 \mathrm{~cm}\left(l_{\mathrm{t}}{ }^{\prime}=1 \mathrm{~mm}, D=0.33 \mathrm{~mm}\right)$, the optical path-length difference can be 13 meters. Thus, we used a narrow-linewidth continuous-wave laser (Verdi V10) with a coherence length exceeding $100 \mathrm{~m}$ in our experiments.

\subsection{Optimal Beam Size of the Incident Light}

Another practical concern is that as a scattering medium becomes thicker, fewer photons can pass through the medium and reach the detector, which reduces the signal-to-noise ratio of measurement. Therefore, how to efficiently deliver light to the target position through the scattering medium remains a critical issue. There are two constrains in our case. First, for biological tissue, the light intensity on the tissue surface is capped by the American National Standards Institute (ANSI) safety limit. Second, the laser power is finite such that the beam diameter cannot be too large, because the sources at the rim will not couple well to the target location. Therefore, there exists an optimal beam diameter that can efficiently deliver light to the target location ${ }^{12}$. 


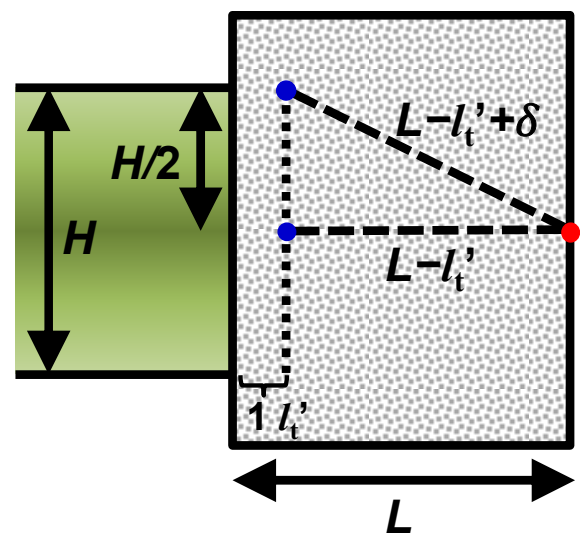

Figure 2. Schematic of the optimal beam diameter to efficiently deliver power to a target location (marked in red).

Figure 2 illustrates how to determine the optimal beam diameter. The target location to deliver light is marked in red, and the incident light is approximated by a line of point sources (marked by the dotted black line) located $1 l_{\mathrm{t}}$ ' below the surface of the medium ${ }^{11}$. When the distance between an off-axis point source and the target point is longer than the distance between the on-axis point source and the target point by $\delta$ (the decay constant of the fluence rate, $=1 / \mu_{\text {eff }}=$ $1 / \sqrt{3 \mu_{\mathrm{a}}\left(\mu_{\mathrm{a}}+\mu_{\mathrm{s}}{ }^{\prime}\right)}$, where $\mu_{\mathrm{a}}$ and $\mu_{\mathrm{s}}{ }^{\prime}$ are the absorption coefficient and the reduced scattering coefficient, respectively), the fluence rate contributed by this off-axis source is only $37 \%$ of the fluence rate contributed by the onaxis source. Considering that the sources located further away from this off-axis point source couple even less power to the target position, we define the optimal beam radius based on this triangle shown in Fig. 2. The analytical expression of the optimal beam diameter $H$ can be worked out using the Pythagorean theorem:

$$
H=2 \sqrt{\left(2 L-2 l_{\mathrm{t}}{ }^{\prime}+\delta\right) \delta} \text {. }
$$

For a sample with thickness $L=25 \mathrm{~mm}, l_{\mathrm{t}}^{\prime}=1 \mathrm{~mm}, \delta=5.74 \mathrm{~mm}$, the optimal beam diameter is $35 \mathrm{~mm}$.

\subsection{Speckle Correlation Time}

To achieve wavefront shaping, it is required to determine the optimum wavefront and shape the incident light before the speckle pattern associated with the scattering medium decorrelates ${ }^{13-15}$. However, even for ex vivo biological tissue, it has been shown that the speckle correlation time decreases as the scattering medium becomes thick ${ }^{16,17}$, due to the Brownian motion of the scatterers. Therefore, a high-speed system that can tolerate the speckle decorrelation is desired. Among all the wavefront shaping techniques, OPC is the fastest, since it determines the optimum wavefront globally based on time reversal, without the need to optimize for each degree of freedom in sequence. Moreover, considering that analog OPC systems based on photorefractive crystals have low energy throughputs, DOPC systems ${ }^{18,19}$ are more suitable to focus light through thick samples.

\subsection{Set-up of our DOPC System}

Based on the previous discussion, we designed a DOPC system schematically shown in Fig. 3. Detailed descriptions of the set-up and the experimental procedures can be found in the reference ${ }^{10}$. Specifically, we used a lens pair composed of L3 and L4 to expand the beam diameter to be $34 \mathrm{~mm}$. Moreover, to avoid exceeding the ANSI safety limit by a focused beam during the playback process, we removed the collecting lens L5, which was used in previous DOPC systems to collect the scattered light and control the speckle size. Furthermore, we intentionally under-sampled the speckle grains during wavefront measurement, which maximized the number of optical degrees of freedom controlled by our DOPC system and improved the focusing quality ${ }^{20}$. Our system runtime, which is the time from the beginning of the wavefront measurement to the appearance of the focus, is $\sim 0.7 \mathrm{~s}$. 


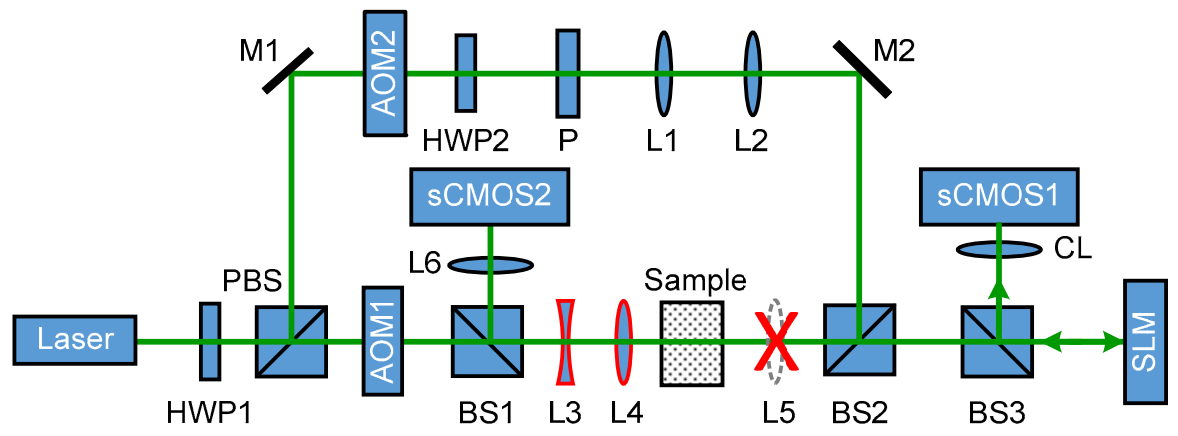

Figure 3. Schematic of the DOPC system. AOM, acousto-optic modulator;BS, beamsplitter; CL, camera lens; HWP, half-wave plate; L, lens; M, mirror; P, polarizer; PBS, polarizing beamsplitter; sCMOS, scientific CMOS camera; SLM, spatial light modulator.

\section{RESULTS}

\subsection{Focusing Light through Chicken Breast Tissue}

We first demonstrated focusing light through centimeter-thick chicken breast tissue. During wavefront measurement, the light intensity on the chicken tissue surface was kept at $200 \mathrm{~mW} / \mathrm{cm}^{2}$, which is the same as the ANSI safety limit.
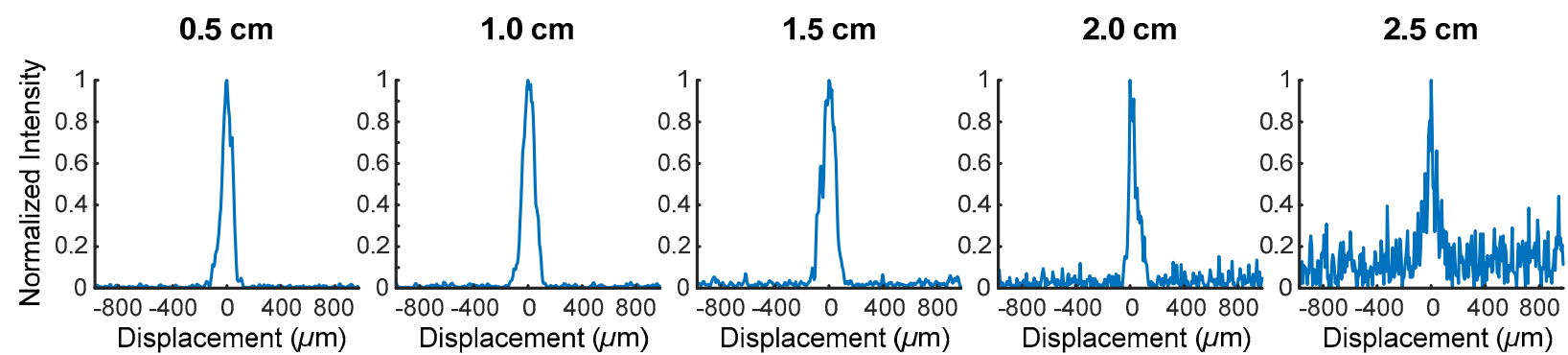

Figure 4. 1D profiles of the OPC foci after light has passed through chicken breast tissue of various thicknesses.

Figure 4 shows the one-dimensional (1D) profiles of the OPC foci after light has passed through chicken tissue samples with thicknesses ranging from $0.5 \mathrm{~cm}$ to $2.5 \mathrm{~cm}$. We can see that with increasing sample thickness, the background fluctuation becomes more and more pronounced. When the sample was $3.0 \mathrm{~cm}$ thick, no focus was observed. The degradation of the focusing quality when the chicken tissue becomes thicker is due to the decreased speckle correlation time $^{10}$.

\subsection{Focusing Light through Tissue-mimcking Phantoms}

The blood vessels in chicken tissue look red, which means they absorb green light strongly. To minimize the absorption of 532-nm light, we used intralipid-gelatin phantoms. The intralipid is shown in Fig. 5(a), which looks very much like milk. The absorption coefficient of intralipid at $532 \mathrm{~nm}$ is $0.07 \mathrm{~cm}^{-1}$, which is close to that of chicken tissue at $800 \mathrm{~nm}$ $\left(0.08 \mathrm{~cm}^{-1}\right)$. The intralipid is even more scattering at $532 \mathrm{~nm}$ than chicken tissue at $800 \mathrm{~nm}$ (reduced scattering coefficient: $10 \mathrm{~cm}^{-1}$ vs. $\left.3.5 \mathrm{~cm}^{-1}\right)^{21}$. So when using this phantom with a green laser, we are mimicking doing experiment with chicken tissue using a near infrared laser. Fig. 5(b) shows two phantoms we used in our experiments, with thicknesses of 96 and $85 \mathrm{~mm}$. A 20-mm-wide cube beamspliter is shown for comparison. In our experiment, the light intensity on the phantom surface was $1.2 \mathrm{~W} / \mathrm{cm}^{2}$, which is six times as high as the ANSI safety limit. However, no damage was observed in the sample after the experiment. 
(a)

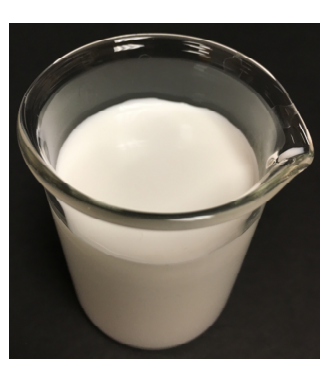

(b)

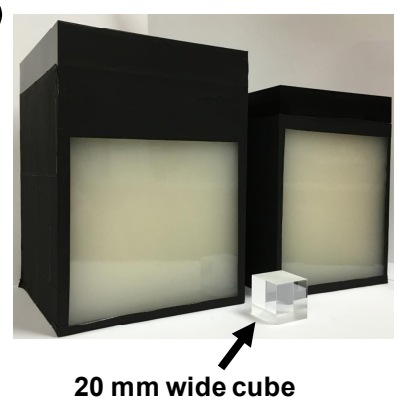

(c)
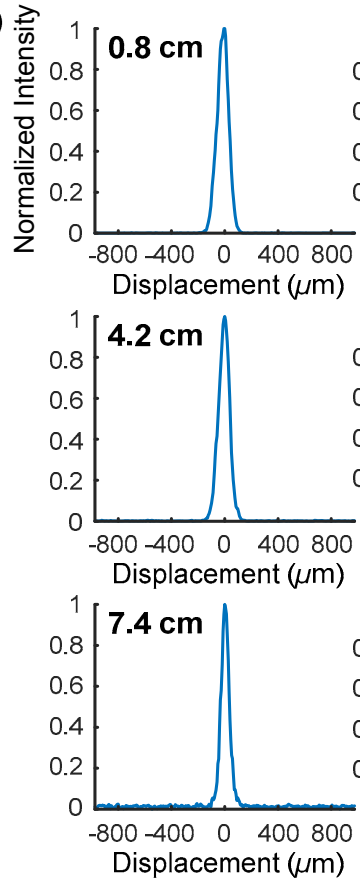
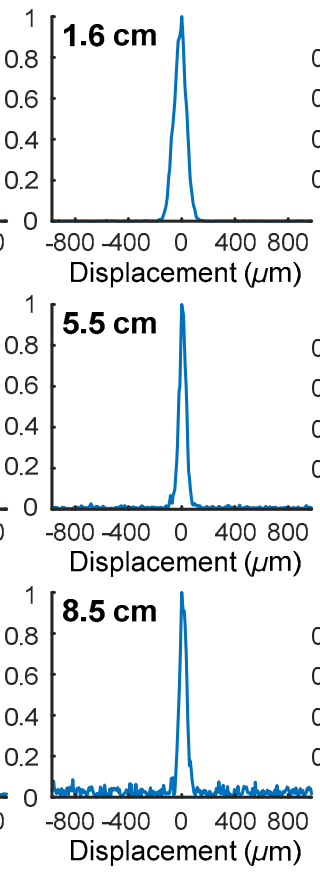
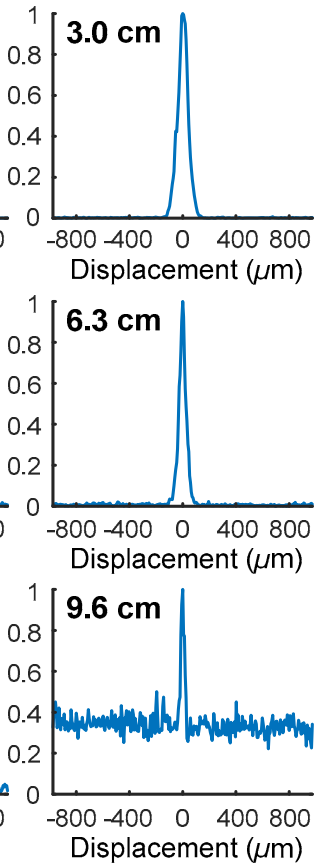

Figure 5. (a) Photo of the intralipid solution. (b) The side view of the intralipid-galetin phantoms with thicknesses of $9.6 \mathrm{~cm}$ and $8.5 \mathrm{~cm}$. (c) 1D profiles of the OPC foci after light has passed through phantoms of various thicknesses.

Figure 5(c) shows the 1D profiles of the OPC foci after light has passed through the phantoms with thicknesses ranging from $0.8 \mathrm{~cm}$ to $9.6 \mathrm{~cm}$. These plots show that we can focus light through a sample even with a thickness of $9.6 \mathrm{~cm}$, although no focus was observed when the thickness was $10.0 \mathrm{~cm}$. Focusing light through a $9.6 \mathrm{~cm}$ thick sample by DOPC is quite remarkable, because the transmitted photons have experienced on average at least 1000 scattering events, and only a tiny portion of the entire scattered wavefront is phase conjugated (The collected light power was only $10^{-9}$ of the incident power on the sample).

\section{CONCLUSIONS}

In conclusion, using an optimized DOPC system, we focused 532-nm light through chicken tissue up to $2.5 \mathrm{~cm}$ thick and through tissue-mimicking phantoms up to $9.6 \mathrm{~cm}$ thick. The $9.6 \mathrm{~cm}\left(\sim 100 l_{\mathrm{t}}{ }^{\prime}\right)$ penetration has never been achieved before by any optical focusing or imaging technique. Our work shows the promise of OPC-based wavefront shaping techniques to revolutionize biomedicine with deep-tissue non-invasive optical imaging, manipulation, and therapy.

\section{ACKNOWLEDGEMENT}

This work was sponsored in part by National Institute of Health grants DP1 EB016986 (NIH Director's Pioneer Award) and R01 CA186567 (NIH Director's Transformative Research Award).

\section{REFERENCES}

1. V. Ntziachristos, "Going deeper than microscopy: the optical imaging frontier in biology," Nat Methods 7(8), 603614 (2010).

2. Y. Liu, C. Zhang and L. V. Wang, "Effects of light scattering on optical-resolution photoacoustic microscopy," $J$ Biomed Opt 17(12), 126014 (2012). 
3. I. M. Vellekoop and A. P. Mosk, "Focusing coherent light through opaque strongly scattering media," Opt Lett 32(16), 2309-2311 (2007).

4. S. Popoff, G. Lerosey, R. Carminati, M. Fink, A. Boccara and S. Gigan, "Measuring the transmission matrix in optics: an approach to the study and control of light propagation in disordered media," Phys Rev Lett 104(10), 100601 (2010).

5. M. Cui, "A high speed wavefront determination method based on spatial frequency modulations for focusing light through random scattering media," Opt Express 19(4), 2989-2995 (2011).

6. Z. Yaqoob, D. Psaltis, M. S. Feld and C. Yang, "Optical phase conjugation for turbidity suppression in biological samples," Nat Photonics 2(2), 110-115 (2008).

7. C. Stockbridge, Y. Lu, J. Moore, S. Hoffman, R. Paxman, K. Toussaint and T. Bifano, "Focusing through dynamic scattering media," Opt Express 20(14), 15086-15092 (2012).

8. E. J. McDowell, M. Cui, I. M. Vellekoop, V. Senekerimyan, Z. Yaqoob and C. Yang, "Turbidity suppression from the ballistic to the diffusive regime in biological tissues using optical phase conjugation," J Biomed Opt 15(2), 025004 (2010).

9. Y. Shen, Y. Liu, C. Ma and L. V. Wang, "Focusing light through scattering media by full-polarization digital optical phase conjugation," Opt Lett 41(6), 1130-1133 (2016).

10. Y. Shen, Y. Liu, C. Ma and L. V. Wang, "Focusing light through biological tissue and tissue-mimicking phantoms up to $9.6 \mathrm{~cm}$ in thickness with digital optical phase conjugation," J Biomed Opt 21(8), 085001 (2016).

11. L. V. Wang and H. Wu, Biomedical optics : principles and imaging, Wiley-Interscience, Hoboken, N.J. (2007).

12. L. V. Wang, R. E. Nordquist and W. R. Chen, "Optimal beam size for light delivery to absorption-enhanced tumors buried in biological tissues and effect of multiple-beam delivery: a Monte Carlo study," Applied Optics 36(31), 8286-8291 (1997).

13. Y. Liu, P. Lai, C. Ma, X. Xu, A. A. Grabar and L. V. Wang, "Optical focusing deep inside dynamic scattering media with near-infrared time-reversed ultrasonically encoded (TRUE) light," Nat Commun 6(5904 (2015).

14. C. Ma, F. Zhou, Y. Liu and L. V. Wang, "Single-exposure optical focusing inside scattering media using binarized time-reversed adapted perturbation," Optica 2(10), 869-876 (2015).

15. Y. Liu, C. Ma, Y. Shen and L. V. Wang, "Bit-efficient, sub-millisecond wavefront measurement using a lock-in camera for time-reversal based optical focusing inside scattering media," Opt Lett 41(7), 1321-1324 (2016).

16. D. J. Pine, D. A. Weitz, P. M. Chaikin and E. Herbolzheimer, "Diffusing wave spectroscopy," Physical Review Letters 60(12), 1134-1137 (1988).

17. J. Brake, M. Jang and C. Yang, "Analyzing the relationship between decorrelation time and tissue thickness in acute rat brain slices using multispeckle diffusing wave spectroscopy," JOSA A 33(2), 270-275 (2016).

18. M. Cui and C. Yang, "Implementation of a digital optical phase conjugation system and its application to study the robustness of turbidity suppression by phase conjugation," Opt Express 18(4), 3444-3455 (2010).

19. C.-L. Hsieh, Y. Pu, R. Grange, G. Laporte and D. Psaltis, "Imaging through turbid layers by scanning the phase conjugated second harmonic radiation from a nanoparticle," Opt Express 18(20), 20723-20731 (2010).

20. Y. Shen, Y. Liu, C. Ma and L. V. Wang, "Sub-Nyquist sampling boosts targeted light transport through opaque scattering media," Optica 4(1), 97-102 (2017).

21. P. Lai, X. Xu and L. V. Wang, "Dependence of optical scattering from Intralipid in gelatin-gel based tissuemimicking phantoms on mixing temperature and time," J Biomed Opt 19(3), 035002 (2014). 